\title{
On the application of the classic Kessler and Berry schemes in Large Eddy Simulation models with a particular emphasis on cloud autoconversion, the onset time of precipitation and droplet evaporation
}

\author{
S. Ghosh, P. R. Jonas \\ Department of Physics, UMIST, Manchester, M60 1QD, UK Fax: +44 1612003941
}

Received: 30 June 1997 / Revised: 5 December 1997 / Accepted: 8 December 1997

\begin{abstract}
Many Large Eddy Simulation (LES) models use the classic Kessler parameterisation either as it is or in a modified form to model the process of cloud water autoconversion into precipitation. The Kessler scheme, being linear, is particularly useful and is computationally straightforward to implement. However, a major limitation with this scheme lies in its inability to predict different autoconversion rates for maritime and continental clouds. In contrast, the Berry formulation overcomes this difficulty, although it is cubic. Due to their different forms, it is difficult to match the two solutions to each other. In this paper we single out the processes of cloud conversion and accretion operating in a deep model cloud and neglect the advection terms for simplicity. This facilitates exact analytical integration and we are able to derive new expressions for the time of onset of precipitation using both the Kessler and Berry formulations. We then discuss the conditions when the two schemes are equivalent. Finally, we also critically examine the process of droplet evaporation within the framework of the classic Kessler scheme. We improve the existing parameterisation with an accurate estimation of the diffusional mass transport of water vapour. We then demonstrate the overall robustness of our calculations by comparing our results with the experimental observations of Beard and Pruppacher, and find excellent agreement.
\end{abstract}

Key words. Atmospheric composition and structure · Cloud physics and chemistry · Pollution - Meteorology and atmospheric dynamics · Precipitation

\section{Introduction}

Representations of microphysical processes that control the partition of water between clouds and precipitation

Correspondence to: S. Ghosh are crucial to the realistic predictions of cloud models including the large eddy simulation (LES) models.

Kessler (1969) pioneered the introduction of the effect of varying updraughts into cloud models. In his approach, all water is first condensed as cloud water, with small drop size (roughly 5-30 $\mu \mathrm{m}$ ) and negligible terminal velocity. Then a process called autoconversion begins. This involves the formation of precipitation particles either by the aggregation of several cloud particles or by the action of giant salt nuclei, or similar processes. Water clouds can persist for a long time without precipitating and various measurements show that cloud water contents $\geq 1 \mathrm{~g} \mathrm{~m}^{-3}$ are usually associated with precipitation (Mason, 1957; Singleton and Smith, 1960).

Two different autoconversion schemes are widely used (Simpson and Wiggert, 1969):

$\frac{\mathrm{d} M}{\mathrm{~d} t}=k_{1}(m-a) \quad \mathrm{g} \mathrm{m}^{-3} \mathrm{~s}^{-1}$,

and

$\frac{\mathrm{d} M}{\mathrm{~d} t}=\frac{m^{2}}{60\left(5+\frac{0.0366 N_{b}}{m D_{b}}\right)} \quad \mathrm{g} \mathrm{m}^{-3} \mathrm{~s}^{-1}$,

where Eq. (1) is due to Kessler (1969) and Eq. (2) to Berry (1968). $M$ and $m$ are the precipitation water content and the cloud water contents, respectively, $k_{1}$ the autoconversion rate $\left(\mathrm{s}^{-1}\right), a$ the autoconversion threshold $\left(\mathrm{g} \mathrm{m}^{-3}\right), N_{b}$ and $D_{b}$ are the droplet number density $\left(\right.$ no. $\left.\mathrm{cm}^{-3}\right)$ and droplet relative dispersion at the cloud base. We shall first discuss the implications of the Kessler formula.

\section{The Kessler autoconversion scheme}

Kessler assumed that the rate of autoconversion increases with the cloud water content but is zero for some values below the threshold $a$, below which cloud conversion does not occur. The parameter $k_{1}$ is the 
reciprocal of the $1 / e$ "conversion time" of the cloud water.

In models which use the Kessler scheme, the conversion of cloud water to precipitation does not start until the cloud content produced in saturated updraughts has exceeded $a$. Thereafter, the cloud water responds to its accretion by relatively large precipitation particles in addition to processes of vertical advection and condensation.

The real atmosphere's analogue to the autoconversion threshold can be height dependent. There abounds considerable arbitrariness with regard to the choice of appropriate values for $k_{1}$ and $a$, although the recommended value for $k_{1}$ by Kessler is $10^{-3}$, actual values of $a$ can only be ascertained from experiments which are often difficult to conduct. In the absence of such supporting experimental data various modellers have used various combinations of values for $k_{1}$ and $a$. In some of our recent LES modelling studies (Jonas and Ghosh, 1997) we modelled a stratocumulus cloud widely studied during the EUCREX (European Cloud and Radiation Experiment) mission 204 of 18 April 1994. We found that with an adopted value of $10^{-4} \mathrm{~s}^{-1}$ for $k_{1}$ when $a$ is unrealistically set at $1.0 \mathrm{~g} \mathrm{~kg}^{-1}$, there was no evidence of precipitation and the liquid water path (LWP) showed a slow increase to the end of the simulation. It should be noted that the microphysical parameterisations are applied at each point and this implies that the average cloud water content may be less that the autoconversion threshold. Only when $a$ was lowered to $0.3 \mathrm{~g} \mathrm{~kg}^{-1}$ was there a gradual decrease in the LWP, indicating the initiation of precipitation in the simulation. In Table 1 we show the maximum layer averaged rain mass for the model stratocumulus cloud after $4 \mathrm{~h}$ of simulation.

From Table 1 we find that the rain mass increases with lower values of $a$ and the simulations correspondingly showed that the cloud liquid water content increased with increasing values of $a$.

The parameter $k_{1}$ is the rate of autoconversion to precipitation of cloud content in excess of the threshold $a$. When $k_{1}$ is large, the approach to steady state is hastened once $m>a$; when $k_{1}$ is small, the cloud content continues to increase longer in the updraughts after precipitation has started (especially if the accretion process is also weak). Decreasing the autoconversion coefficient $k_{1}$ affects the precipitation development in much the same way as does increasing the conversion threshold $a$. This is clear from Kessler (1969, Figs. 12.7 and 12.8) where the cloud and precipitation profiles corresponding to $k_{1}=10^{-3}$ and $10^{-5}$ in Fig. 12.8 of that

Table 1. Rainmass with varying autoconversion thresholds in a simulated stratocumulus cloud from Jonas and Ghosh (1997)

\begin{tabular}{ll}
\hline$a \mathrm{~g} \mathrm{~kg}^{-1}$ & rainmass $\mathrm{g} \mathrm{kg}^{-1}$ \\
\hline 1.0 & 0.0 \\
0.3 & $1.810^{-3}$ \\
0.2 & $2.510^{-3}$ \\
\hline
\end{tabular}

paper are virtually identical with $a=0.5$ and 2.0 in Fig. 12.7

From the preceding paragraphs it is clear that Kessler's treatment of the autoconversion process, although very useful and amenable to incorporation into cloud models straightforwardly, is rather intuitive. Berry (1968) on the other hand has treated the process more rigorously, and this we discuss in the next section.

\section{The Berry autoconversion scheme}

Berry's equation [Eq. (2)] is developed theoretically from a model of initial cloud growth by condensation and coalescence of cloud-sized particles with each other. The early droplet spectrum near the cloud base has a number concentration of $N_{b}$ drops $\mathrm{cm}^{-3}$ and a relative dispersion $D_{b}$ determined by the condensation nucleus spectrum. The relative dispersion is defined as

$D_{b}=\frac{\sigma_{a}}{\bar{a}}$

where $\sigma_{a}$ is the standard deviation of the droplet radii and $\bar{a}$ is the mean. Equation (2) corresponds to a choice of the $200-\mu \mathrm{m}$ boundary between cloud and precipitation drops. This is realistic for the following reasons:

1. A drop of $200 \mu \mathrm{m}$ diameter has a terminal velocity of 1-2 $\mathrm{m} \mathrm{s}^{-1}$ and is thus beginning to fall at a speed comparable to cumulus updraughts. It can survive a fall of several hundred metres in subsaturated air without complete evaporation.

2. Most $10-\mathrm{cm}$ radars begin to show an echo of a cloud when numerous drops of about this size are present (Simpson and Wiggert, 1969).

Jonas and Mason (1974) studied the evolution of droplet spectra by the combined effects of condensation and coalescence in cumulus clouds. Their computed spectra closely resemble measured spectra and reproduce the bimodal structure observed by Warner (1969). Their study reveals that the onset of precipitation in continental clouds containing a high cloud condensation nucleus concentration depends on the values used for the collection efficiency. Due to enhanced turbulence the growth rate of larger drops would increase significantly.

The work of Jonas and Mason (1974) further validates the results of Berry (1968). Berry (1968) finds that the initial droplet distribution generated just above the cloud base by condensation in a warm cumulus cloud sets the stage for future droplet growth by collection. If these droplets are large or the spread of the distribution broad, the rate at which precipitation droplets will be formed is fast. In a cloud which has a limited lifetime governed by the mesoscale circulation this initial rate of droplet growth may make the difference between rain and no rain.

Twenty-eight typical, condensation-produced spectra were used to cover a range of droplet number and relative dispersion. The subsequent growths were found to fit a pattern of two distinct regions - initial and final, with the boundary between the two regimes at a radius 
of $40 \mu \mathrm{m}-$ an approximate radius of those droplets which contain most of the liquid water (Berry, 1968).

In the initial growth region, the elapsed time ' $T$ ', required for the drops to attain a radius of $40 \mu \mathrm{m}$, excluding the effects of collection, has been calculated by Berry (1968). The flux of cloud water passing $r=40 \mu \mathrm{m}$ averaged over many parcels at various stages of development is just the cloud water content of the parcel $L_{c}$ divided by the time ' $T$ ', which is the autoconversion rate given in Eq. (2).

Upon examining Eqs. (1) and (2) it becomes clear that Berry's (1968) formula, although empirical, accounts for the important microphysical parameters like the droplet number density and the relative dispersion, whereas the Kessler scheme does not. Other than by comparison of the predictions of a cloud model with field experiments there can be no direct way to link the values of $k_{1}$ and $a$ in the Kessler formula in Eq. (1) with the microphysical parameters of the cloud.

The Kessler formula is linear in $m$ while the Berry formula is cubic; as a result the Kessler scheme is more straightforward to use in LES models. Since both Eqs. (1) and (2) represent the same physical process, one way to link the value of $k_{1}$ and/or $a$ in Eq. (1) with microphysical parameters would be to use Eq. (2) to optimise the choice of $k_{1}$ and $a$ for a particular simulation. In this way one would be able indirectly to incorporate the effects of the number density and the relative dispersion without altering the basic structure of the Kessler scheme used within the framework of an LES model.

An important feature of Berry's equation (2) is that a different autoconversion rate is predicted for maritime and for continental clouds. Typically for maritime clouds $N_{b} \sim 50 \mathrm{~cm}^{-3}, D_{b} \sim 0.366$ and for a continental cloud $N_{b} \sim 2000 \mathrm{~cm}^{-3}, D_{b} \sim 0.146$ (Simpson and Wiggert, 1969). The stratocumulus cloud studied extensively during the EUCREX mission [and also by us in our LES modelling (Joans and Ghosh, 1997)] has the number

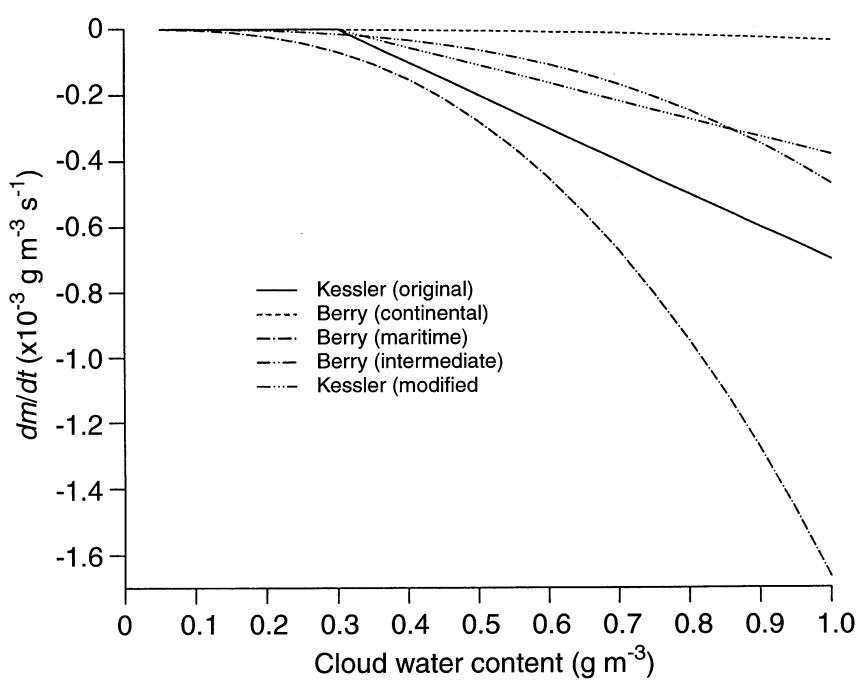

Fig. 1. Rate of change of cloud water content calculated from the classic Kessler (1969) and Berry (1968) schemes density and the relative dispersion in between the maritime and the continental extremes. For this cloud $N_{b} \sim 200 \mathrm{~cm}^{-3}, D_{b} \sim 0.24$ (Pawlowska and Brenguier, 1996), suggesting that although the number concentration is not as high as that of a continental cloud, it had at some stage of its development passed over pollution sources. These features are illustrated in Fig. 1, where we have shown the rate of change of cloud water content $(\mathrm{d} m / \mathrm{d} t)$ as a function of $m$ for both the Kessler and the Berry formulations. In the unmodified Kessler formulation [shown as Kessler (original)] $k_{1}=10^{-3} \mathrm{~s}^{-1}$ and this is more representative of a maritime cloud. This is clearly evident when we compare this graph with the graph corresponding to the Berry (maritime) case. Figure 1 clearly illustrates that the autoconversion rates of maritime clouds are vastly different from those of continental clouds. We also show the rate of change of the cloud water amount for a cloud with microphysical properties in between these limits [Berry (intermediate)]. We have used the Berry scheme directly as well as the optimised Kessler scheme with $k_{1}=5.46 \times 10^{-4}$, where it now becomes clear that with this procedure one can achieve comparable results from both schemes.

One of the important physical parameters that could possibly link the two parameterisation schemes is the time of onset of precipitation. This is discussed in the following section, where we also discuss the procedure for optimising the Kessler scheme in conjunction with the Berry scheme.

\section{On the onset of precipitation of a uniform cloud}

We consider cloud conversion and accretion operating in a deep model cloud of water content $m$ and neglect the advection terms for the sake of simplicity. Changes in cloud and precipitation water content then arise as a result of autoconversion and collection of cloud drops by precipitation.

\subsection{The Kessler formulation}

The equation describing the amount of cloud water can be expressed as

$\frac{\mathrm{d} M}{\mathrm{~d} t}=-\frac{\mathrm{d} m}{\mathrm{~d} t}=k_{1}(m-a)+k_{2}^{\prime} m M^{7 / 8}$

where $k_{1}>0$ when $m>a$ and $k_{2}^{\prime}>0$. The constant $k_{2}^{\prime}=k_{2} E N_{0}^{1 / 8}$ where $E$ is the collection efficiency and $k_{2}=6.96 \times 10^{-4}$ (Kessler, 1969).

Since there is no vertical motion we also require that

$M+m=m_{0}, \quad 0 \leq t \leq \infty$,

where $m_{0}$ is the amount of cloud water when $t=0$ and since

$M^{7 / 8} \simeq M$

$\frac{\mathrm{d} m}{\mathrm{~d} t}=k_{2}^{\prime} m^{2}-\left(k_{1}+k_{2}^{\prime} m_{0}\right) m+k_{1} a$, 
or,

$t=\int_{m_{0}}^{m} \frac{\mathrm{d} m}{k_{2}^{\prime} m^{2}-\left(k_{1}+k_{2}^{\prime} m_{0}\right) m+k_{1} a}$.

Integration of this equation will give us a handle on the time of onset of precipitation. A convenient measure would be to evaluate the time over which $m$ reduces from $m_{0}$ to $m_{0} / e$ (this can be easily obtained from plots of $m$ as a function of $t$ ).

In order to integrate Eq. (8) we set

$k_{2}^{\prime}=C$,

$-\left(k_{1}+k_{2}^{\prime} m_{0}\right)=2 B$

$k_{1} a=A$,

and we have

$t=-\left[\frac{1}{\sqrt{A C-B^{2}}} \tan ^{-1} \frac{c m+B}{\sqrt{A C-B^{2}}}\right]_{m}^{m_{0}}$

when

$A C>B^{2}$

or

$t=-\frac{1}{2 \sqrt{B^{2}-A C}} \ln \left[\frac{C m+B-\sqrt{B^{2}-A C}}{C m+B-\sqrt{B^{2}-A C}}\right]_{m}^{m_{0}}$

when

$B^{2}>A C$.

From the two new solutions given by Eqs. (9) and (11) it is clear that the times of onset of precipitation will vary according to the conditions imposed by Eqs. (10) and (12) which are functions of the autoconversion coefficient $k_{1}$, the autoconversion threshold $a$, the initial cloud water amount $m_{0}$ and the accretion coefficient $k_{2}^{\prime}$.

In Fig. 2a we examine the effect of the initial cloud water content with fixed values of the autoconversion coefficient $\left(k_{1}=5.45 \times 10^{-4} \mathrm{~s}^{-1}\right)$ and threshold $\left(a=0.3 \mathrm{~g} \mathrm{~m}^{-3}\right)$. We find that with lower initial cloud water content the onset times are longer. Note that the kinks in the curves show the region when $m=a$, below which point only the process of accretion is operative.

In Fig. $2 b$ we fix $m_{0}$ at $1 \mathrm{~g} \mathrm{~m}^{-3}$ and $a$ at $0.3 \mathrm{~g} \mathrm{~m}^{-3}$ and examine the effect of varying $k_{1}$. For low values of $k_{1}$, the precipitation onset times are large as is expected. In Fig. 2c we examine the effect of varying the autoconversion threshold and find that the onset times are higher for larger threshold values.

\subsection{The Berry formulation}

In the Berry formulation (Berry, 1968) the equation equivalent to Eq. (4) is

$\frac{\mathrm{d} M}{\mathrm{~d} t}=-\frac{\mathrm{d} m}{\mathrm{~d} t}=\frac{m^{2}}{60\left(5+\frac{0.0366 N_{b}}{m D_{b}}\right)}+k_{2}^{\prime} m M^{7 / 8}$.
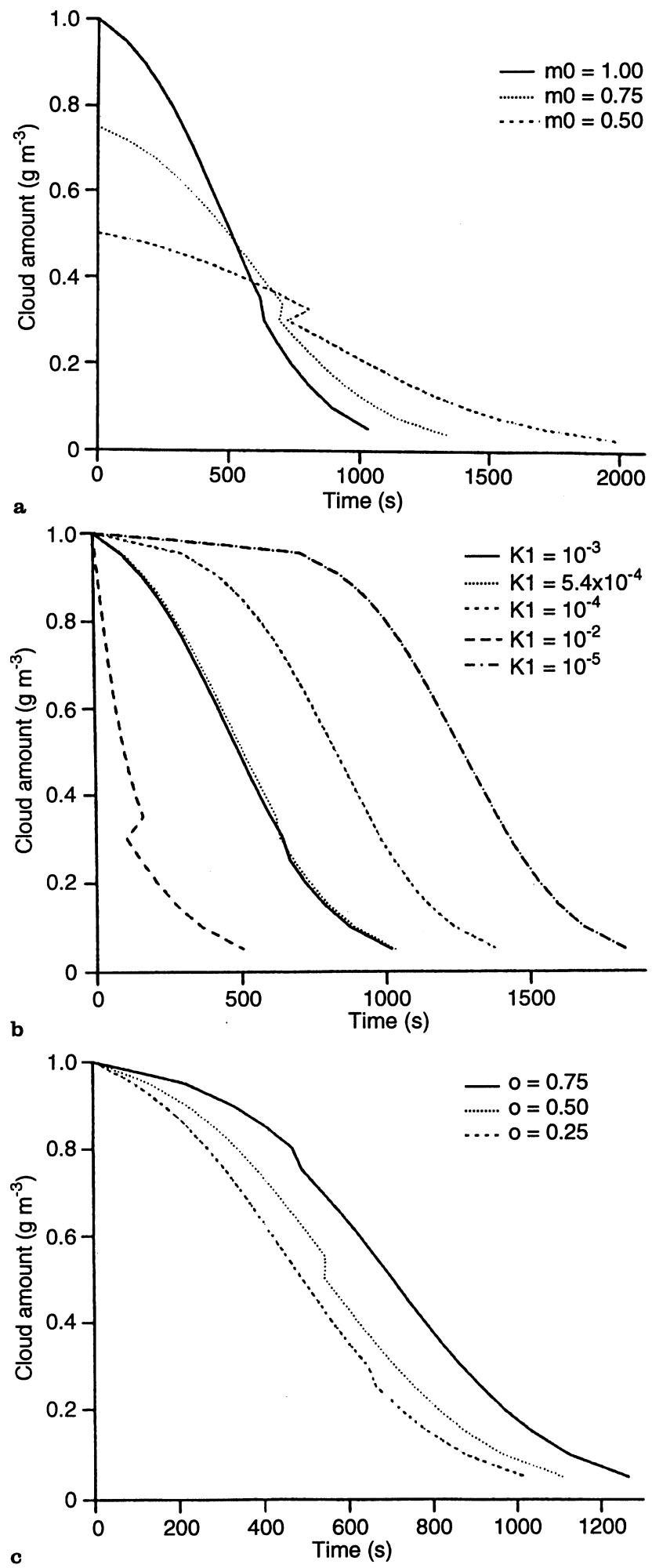

Fig. 2a-c. Time of onset of precipitation calculated from the analytical solutions of Eqs. (9) and (11) using the Kessler (1969) scheme. a Effect of varying initial cloud water content with fixed autoconversion coefficient and threshold. b Effect of varying autoconversion coefficient with fixed initial cloud water content and autoconversion threshold. c Effect of varying autoconversion threshold with fixed initial cloud water content and autoconversion coefficient 
Typically, $\quad N_{b} \sim 200 \mathrm{~cm}^{-3}, \quad m \sim 0.5 \mathrm{~g} \mathrm{~m}^{-3}, \quad$ and $D_{b} \sim 0.24$ and for this case $\frac{0.0366 N_{b}}{m D_{b}} \gg 5.0$, and therefore approximating Eq. (13) we can write

$\frac{\mathrm{d} M}{\mathrm{~d} t}=-\frac{\mathrm{d} m}{\mathrm{~d} t} \simeq k_{1} m^{3}+k_{2}^{\prime} m M$

where $k_{1}=60 \times 0.0366 N_{b} / D_{b}$.

Hence,

$t=-\int_{m+0}^{m} \frac{\mathrm{d} m}{k_{1} m^{3}-k_{2}^{\prime} m^{2}+k_{2}^{\prime} m m_{0}}$.

Further by setting

$$
\begin{aligned}
& k_{2}^{\prime} m_{0}=A^{\prime}, \\
& -k_{2}^{\prime}=2 B^{\prime}, \\
& k_{1}=C^{\prime}
\end{aligned}
$$

we obtain for the case when $B^{\prime 2}>A^{\prime} C^{\prime}$

$$
\begin{aligned}
t= & \frac{1}{A^{\prime}}[\ln (m)]_{m}^{m_{0}}-\frac{1}{2 A^{\prime}} \ln \left[A^{\prime}+2 B^{\prime} m+C^{\prime} m^{2}\right]_{m}^{m_{0}} \\
& -\frac{B^{\prime}}{2 A^{\prime} \sqrt{B^{\prime 2}-A^{\prime} C^{\prime}}} \\
& \times \ln \left[\frac{C^{\prime} m+B^{\prime}-\sqrt{B^{\prime 2}-A^{\prime} C^{\prime}}}{C^{\prime} m+B^{\prime}+\sqrt{B^{\prime 2}-A^{\prime} C^{\prime}}}\right]_{m}^{m_{0}} .
\end{aligned}
$$

As an illustrative example, as we have seen before, for the EUCREX stratocumulus cloud, $N_{b} \sim 200 \mathrm{~cm}^{-3}$, $D_{b}=0.24 ;$ this yields $k_{1}=5.46 \times 10^{-4} \mathrm{~s}^{-1}$. Setting $N_{0}=10^{7}, E=1.0$, we obtain $k_{2}=5.22 \times 10^{-3}$. For an initial cloud water content of $1 \mathrm{~g} \mathrm{~m}^{-3}$, we find that $A^{\prime} C^{\prime}<B^{\prime 2}$ and therefore indeed Eq. (16) is valid.

In contrast for typical maritime clouds $N_{b} \sim 10 \mathrm{~cm}^{-3}$, $D_{b} \sim 0.4$, yielding $k_{1}=1.82 \times 10^{-2} \mathrm{~s}^{-1}$. For this situation $A^{\prime} C^{\prime}>B^{\prime 2}$ and we obtain a different solution:

$$
\begin{aligned}
t= & \frac{1}{A^{\prime}}[\ln (m)]_{m}^{m_{0}}-\frac{1}{2 A^{\prime}} \ln \left[A^{\prime}+2 B^{\prime} m+C^{\prime} m^{2}\right]_{m}^{m_{0}} \\
& -\frac{B^{\prime}}{2 A^{\prime} \sqrt{B^{\prime 2}-A^{\prime} C^{\prime}}} \tan ^{-1}\left[\frac{C^{\prime} m+B^{\prime}}{\sqrt{A^{\prime} C^{\prime}-B^{\prime 2}}}\right]_{m}^{m_{0}} .
\end{aligned}
$$

Equations (16) and (17) are also two new solutions and predict different onset times for microphysical parameters appropriate for continental and maritime clouds and also provide useful insights on the relative importance of the processes of autoconversion and accretion.

In Fig. 3a we show cloud water content as a function of time for a typical continental cloud, a maritime cloud, and a cloud with microphysical properties in between these two extremes such as the EUCREX stratocumulus. The curves are obtained from the solutions in Eqs. (16) and (17) and show that the onset times of precipitation for a continental cloud is $\sim 1200 \mathrm{~s}$ as compared to $600 \mathrm{~s}$ for a maritime cloud.

In Fig. $3 b$ we show the effect of initial cloud water content with $N_{b} \sim 200 \mathrm{~cm}^{-3}$ and $D_{b} \sim 0.24$. Larger initial cloud water content yields smaller onset times. This trend is also observed from the Kessler formulation (see Fig. 2a).
One of the objectives of this analysis is to establish an equivalence between the classic Kessler parameterisation and the Berry (1968) parameterisation. Although the Berry (1968) parameterisation is able to differentiate between maritime and continental clouds, it is not as widely used in many LES models. Most bulk-parameterised cloud models continue to utilise some modified version of the Kessler scheme [for example Rutledge and Hobbs (1983); the widely used U.K. Meteorological Office LES model (Derbyshire et al., 1994)].

With the present analysis we are now in a position judiciously to specify the autoconversion coefficient and the threshold in any cloud model using the Kessler scheme rather than use arbitrary values. We suggest that when cloud microphysical observations are available, the observed values should be used as far as possible for the purposes of model constrainment. Then we recommend the use of the Berry (1968) formulation, Eq. (2), to calculate the autoconversion rate. Assuming that Eqs. (1) and (2) are identical rates, one can now
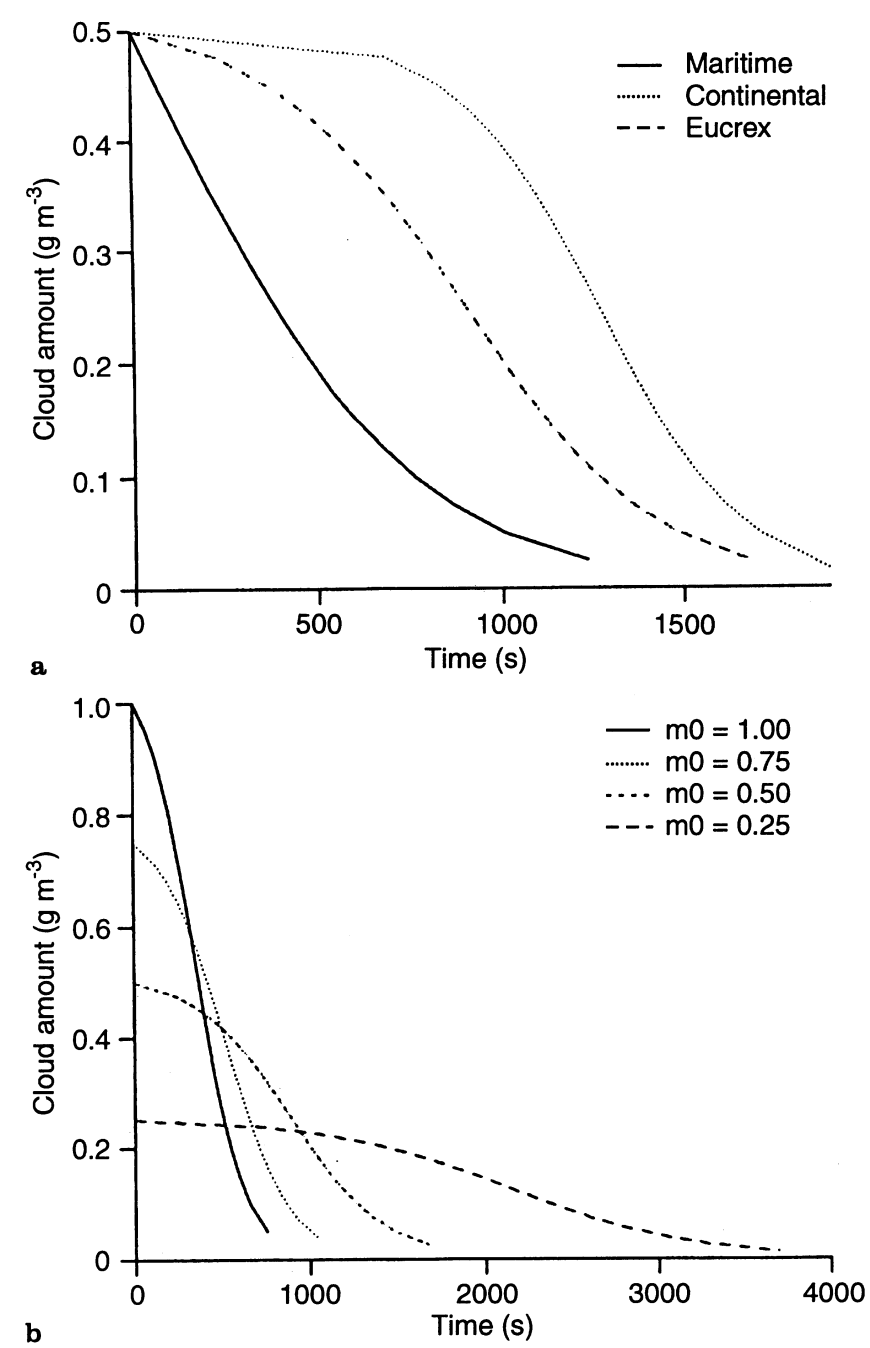

Fig. 3a, b. Time of onset of precipitation calculated from the analytical solutions of Eqs. (16) and (17) using the Berry (1968) scheme. a Cloud water content for different cloud types. b Effect of varying initial cloud water content with $N_{b} \sim 200 \mathrm{~cm}^{-3}, D_{b} \sim 0.24$ 
calculate $k_{1}$ with a prescribed value of $a$, or vice versa. In this way we need to specify one rather than two adjustable parameters.

For example if we assume that $a=0.33 \mathrm{~kg} \mathrm{~m}^{-3}$ then we obtain a value of $k_{1}=5.46 \times 10^{-4} \mathrm{~s}^{-1}$ as the correct input to the Kessler scheme. This enables one also to ascertain the conditions under which the two schemes can yield nearly identical cloud water contents. We show this in Fig. 4, where we obtain nearly the same cloud water content as a function of time. Using this approach we find significant improvements in the predictive capacities of our LES modelling of the EUCREX stratocumulus cloud.

In this context it is worth mentioning that Weinstein (1970) showed from a sensitivity analysis that the autoconversion threshold $a$ is a crucial parameter. Rutledge and Hobbs (1983) deduced a value of $7 \times 10^{-4} \mathrm{~kg} \mathrm{~kg}^{-1} \sim 0.56 \times 10^{-4} \mathrm{~kg} \mathrm{~m}^{-3}$ from airborne observations of cloud and rainwater contents in warm stratiform clouds. From the procedure just discussed with the above value of $a$ the calculated value of $k_{1}$ to be used in a cloud model involving the Kessler scheme turns out to be $1.08 \times 10^{-3} \mathrm{~s}^{-1}$, very close to the value of $1.00 \times 10^{-3} \mathrm{~s}^{-1}$ used by Rutledge and Hobbs (1983). This example demonstrates the overall simplicity of the outlined procedure.

In the next section we examine the process of raindrop evaporation within the framework of the Kessler scheme and include an accurate estimation of the diffusion coefficient of water vapour used in the scheme.

\section{Improved parameterisation for the evaporation of raindrops with an accurate estimation of diffusional mass transport of water vapour}

In order to account for the rate of change of mass of an evaporating raindrop one finds that various paramete-

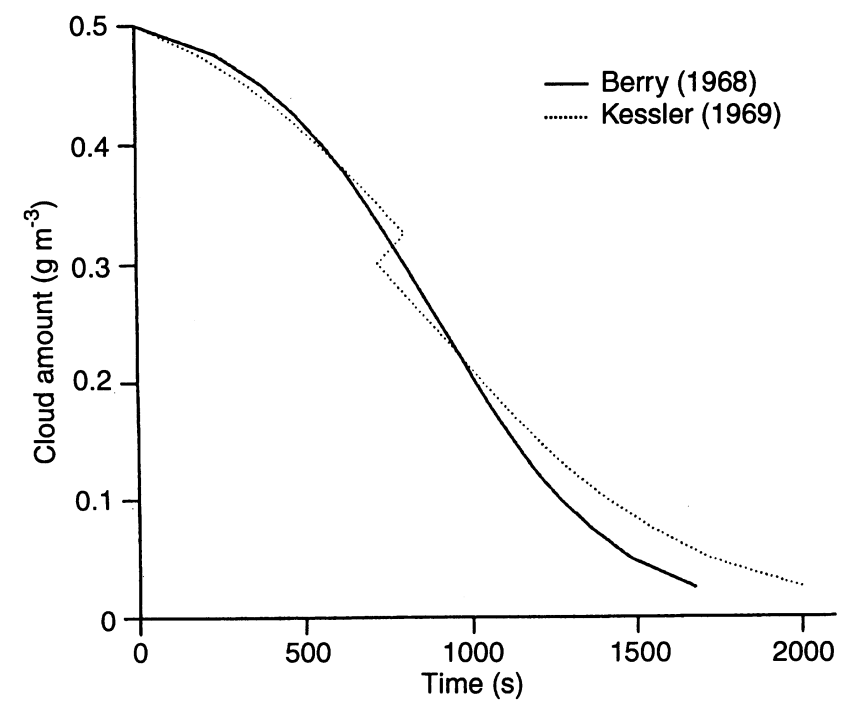

Fig. 4. Equivalence of the optimised Kessler (1969) scheme and the Berry (1968) scheme for $N_{b} \sim 200 \mathrm{~cm}^{-3}, D_{b} \sim 0.24$ rised expressions are frequently used in numerical models, particularly in LES models. Many of these models incorporate the calculations of Kessler (1969). A careful evaluation of this scheme reveals that some of the parameterisations involved with the quantification of the droplet evaporation term are directly dependent on the diffusion coefficient of water vapour. Modellers use diffusion data reported under laboratory conditions which are often different from atmospheric conditions. Secondly, many models, and in particular some of the LES models ignore the temperature difference between the evaporating droplet and the environmental air. In this paper we re-evaluate the evaporation term involved in the Kessler (1969) scheme by calculating the exact value of the diffusion coefficient of water vapour (accounting also for the finite dipole moment of water vapour) and taking account of the temperature difference between the drop and the ambient air. We compare our results with the observations of Kinzer and Gunn (1951), with Kessler (1969), and also with the more recent and definitive observations of Beard and Pruppacher (1971). Significant improvements are obtained by using our approach and our results agree very well with the observations of Beard and Pruppacher (1971).

\subsection{On the evaporation of a rain droplet}

For an evaporating drop, if one assumes that over a limited range the saturation vapour density at the surface of the drop is a linear function of the absolute temperature, then

$\rho_{a}-\rho_{b} \simeq \rho_{b}\left(\frac{1-U}{U}\right)-\left(T_{b}-T_{a}\right)\left(\frac{\mathrm{d} \rho}{\mathrm{d} T}\right)_{s}$,

where $U$ is the fractional relative humidity and $(\mathrm{d} \rho / \mathrm{d} T)_{s}$ is the mean slope of the saturation vapour densitytemperature curve determined at the temperature of the drop and the environment, $\rho_{a}$ and $\rho_{b}$ are the saturation density of water vapour at the temperature of the drop, and the density of water vapour in the environmental air (Kinzer and Gunn, 1951).

Also, since

$U=\frac{\rho_{b}}{\rho_{a}}$,

$\rho_{a}-\rho_{b} \simeq \rho_{a}(1-U)-\left(T_{b}-T_{a}\right)\left(\frac{\mathrm{d} \rho}{\mathrm{d} T}\right)_{s}$.

In Eqs. (18) and (20), $\left(T_{b}-T_{a}\right)$ is the temperature difference between the evaporating drop and the environment. From the experimental observations of Kinzer and Gunn (1951), it was found that the temperature of the freely falling drops are very close to the wet-bulb temperature.

Equation (20) can be used to estimate the vapour density difference from which the evaporation rate is determined only when

$\left(T_{b}-T_{a}\right)\left(\frac{\mathrm{d} \rho}{\mathrm{d} T}\right)_{s}<\rho_{a}(1-U)$. 
From the measurements of the variation of saturation density of water vapour (Mason, 1957; Smithsonian Tables, 1958) it is clear that $(\mathrm{d} \rho / \mathrm{d} T)_{s}$ increases rapidly with increasing temperatures. Using representative values of the relevant quantities we find that Eq. (21) is true for temperatures colder than the freezing point of water. In this study we are mainly concerned with layer clouds without ice.

From a record of the temperature measurements of a stratocumulus cloud studied during the EUCREX measurements (Pawlowska and Brenguier, 1996), it is found that the temperature within the cloud ranged between $-0.5^{\circ} \mathrm{C}$ and $0{ }^{\circ} \mathrm{C}$. From a summary of observations on non-freezing layer clouds in mid-latitudes, it is known that drizzle may often fall from clouds which contain no ice crystals and is therefore produced by coalescence of droplets. For supercooled clouds in which no ice crystals were detected, about $30 \%$ were colder than $-12{ }^{\circ} \mathrm{C}$, only $12 \%$ colder than $-16^{\circ} \mathrm{C}$, and only $5 \%$ colder than: $-20^{\circ} \mathrm{C}$. Continuous precipitation can be maintained only if the cloud water is replenished by a persistent vertical motion fed, perhaps over a large region, by low-level convergence in the wind field (Mason, 1957).

From the preceding paragraph we find that for a vast majority of layer clouds over the mid-latitudes, we must be concerned with temperatures ranging from $0{ }^{\circ} \mathrm{C}$ to about $-10^{\circ} \mathrm{C}$, a temperature range safely applicable to use Eq. (20).

Some of the LES codes currently in use, including the U.K. Meteorological Office LES code (Derbyshire et al., 1994) use the Kessler (1969) scheme for parameterising the evaporation rate of precipitation, and this is based on the measurements of Kinzer and Gunn (1951). These authors, however, report measurements covering a temperature range of $0-40^{\circ} \mathrm{C}$. Hence, using the Kinzer and Gunn (1951) measurements to derive empirical parameterisations for the raindrop evaporation calculations at low temperatures (particularly sub-zero temperatures) is likely to yield inaccurate results. In fact, Kessler (1969) points out that his estimation of $D_{v}\left(\rho_{b}-\rho_{a}\right)$ (where $D_{v}$ is the diffusivity of water vapour in air) could involve an error bar as large as $40 \%$. It is possible that this error can be greatly reduced if the term $D\left(\rho_{b}-\rho_{a}\right)$ is estimated as accurately as possible.

We shall first estimate the magnitude of the temperature difference between the drop and the ambient air, for the conditions typical to stratocumulus clouds observed over the mid-latitudes. For the EUCREX observations of 18 April 1994, the relative humidity was $\sim 70 \%$.

Assuming $T_{b}-T_{a} \simeq T_{b}-T_{w}$, where $T_{w}$ is the wetbulb temperature, and neglecting the weak dependence of $L$ (latent heat of condensation) on the temperature (Rogers and Yau, 1989),

$T_{b}-T_{w}=\frac{L}{C_{p}}\left(W_{s}-W\right)$,

where $W$ and $W_{s}$ are the water vapour mixing ratio and the saturation mixing ratio, respectively.
We can also write,

$T_{b}-T_{w}=\frac{L}{C_{p}} W_{s}(1-U)$.

Typically $\frac{L}{C_{p}} \sim 2.5 \times 10^{3} \mathrm{~K}$, and for $U=0.7$ and $W_{s}=4 \times 10^{-3} \mathrm{~kg} \mathrm{~kg}^{-1}$ between $800-900 \mathrm{mb}$ and at temperatures between $0-4{ }^{\circ} \mathrm{C}$, we find that $T_{b}-T_{w} \sim 3^{\circ} \mathrm{C}$. This temperature difference is often not accounted for in many of the LES codes. Neglecting the temperature difference, we have the following approximate relationship:

$\rho_{a}-\rho_{b} \simeq \rho_{a}(1-U)$.

However, as we have seen earlier, in cold and humid conditions, a temperature difference of $\sim 3^{\circ} \mathrm{C}$ can contribute significantly to the saturation vapour density difference at the surface of the drop, and it is more appropriate not to assume that the drop is at the same temperature as the environment. In Table 2 we show the saturated vapour density difference at varying temperatures including the contribution of the temperature difference using Eq. (20) and compare this with the case when the temperature difference is neglected [using the approximate Eq. (24)]. In this table we show results corresponding to a relative humidity of $70 \%$. The last column of this table shows the percentage error that would be encountered if the temperature difference is not accounted for.

From Table 2 we find that at a relative humidity of $70 \%$ and within a temperature range of $-10^{\circ} \mathrm{C}$ to $5^{\circ} \mathrm{C}$, a typical temperature range encountered in many clouds, it is very important to account for the temperature difference between the evaporating droplet and the environment.

The rate of change of mass of a freely falling water drop in $\mathrm{g} \mathrm{s}^{-1}$ is given by

$\frac{\delta M_{i}}{\delta t}=\left[4 \pi a\left(1+\frac{F}{s}\right)\right]\left[D_{v}\left(\rho_{a}-\rho_{b}\right)\right]$,

where $a$ is the drop radius, $s$ is the equivalent thickness of the transition shell outside the drop, $F$, a dimensionless factor, and $D_{v}$ the diffusion coefficient. Kinzer and Gunn's tabulation of the first term shows that this term is nearly independent of the ambient temperature and humidity and can be fitted to an accuracy of about $\pm 20 \%$ (Kessler, 1969) by:

$4 \pi a\left(1+\frac{F}{s}\right)=2.24 \times 10^{3} D^{8 / 5}$,

Table 2 Saturated water vapour density difference as a function of temperature

\begin{tabular}{|c|c|c|c|c|}
\hline $\begin{array}{l}T, \\
{ }^{\circ} \mathrm{C}\end{array}$ & $\begin{array}{l}\left(T_{b}-T_{a}\right), \\
{ }^{\circ} \mathrm{C}\end{array}$ & $\begin{array}{l}\left(\rho_{a}-\rho_{b}\right) \\
\text { [Eq. }(20)]\end{array}$ & $\begin{array}{l}\left(\rho_{a}-\rho_{b}\right) \\
\text { [Eq. (24)] }\end{array}$ & $\%$ error \\
\hline-10 & 1.345 & 0.5346 & 0.7074 & 24.42 \\
\hline-5 & 1.983 & 0.6648 & 1.0221 & 34.96 \\
\hline 0 & 2.879 & 0.7375 & 1.4541 & 49.28 \\
\hline 5 & 4.121 & 0.6423 & 2.0391 & 68.50 \\
\hline
\end{tabular}


where $D=2 a$ is the drop diameter in metres.

According to Kessler (1969), the second term is related linearly to the saturation deficit $\alpha$ as

$D_{v}\left(\rho_{a}-\rho_{b}\right)=10^{-5} \alpha\left[\mathrm{g} \mathrm{m}^{-1} \mathrm{~s}^{-1}\right]$.

Since the first term in square brackets in Eq. (25) is independent of the temperature and the humidity, the parameterisation in Eq. (26) is adequately represented. However, the second term in square brackets in Eq. (25) does strongly depend upon the temperature and the relative humidity. Hence it is very important to parameterise this term as accurately as possible and preferably in a manner that can allow one to incorporate the temperature dependence.

Using Eq. (24) we can write

$D_{v}\left(\rho_{a}-\rho_{b}\right) \simeq D_{v} \rho_{a}(1-U)$.

When one compares Eqs. (27) and (28), it is found that in order for Eq. (27) to be dimensionally correct, the right-hand side of Eq. (27) should involve a factor that should have the units of a diffusion coefficient $\left(L^{2} T^{-1}\right)$. Indeed, the factor $10^{-5}$ on the right-hand side should have units of $D_{v}$, and this is possible when $D_{v}=0.1 \mathrm{~cm}^{2} \mathrm{~s}^{-1}$. Or, in other words, the Kessler (1969) scheme as described in Eq. (27) implicitly assumes that the diffusion coefficient of water vapour in air is equal to $0.1 \mathrm{~cm}^{2} \mathrm{~s}^{-1}$. However, the observed values of the diffusion coefficient of water vapour in air are much higher. For example, at $0{ }^{\circ} \mathrm{C} D_{v}=0.2 \mathrm{~cm}^{2} \mathrm{~s}^{-1}$ (Smithsonian Tables, 1958). Assuming a value of $0.1 \mathrm{~cm}^{2} \mathrm{~s}^{-1}$ would therefore at the very least underpredict the evaporation rate by a factor of 2 .

However, the most accurate procedure is to use Eq. (20) and one now obtains:

$D_{v}\left(\rho_{a}-\rho_{b}\right) \simeq D_{v}\left[\rho_{a}(1-U)-\left(T_{b}-T_{a}\right)\left(\frac{\mathrm{d} \rho}{\mathrm{d} T}\right)_{s}\right]$.

Thus, an immediate and obvious improvement to the Kessler (1969) microphysical scheme would be to use Eq. (29) instead of Eq. (27). Experimental values of diffusion coefficients are usually reported for laboratory conditions only, and modellers are often constrained by the availability of diffusion coefficient data relevant to the real atmosphere, particularly as a function of altitude. Hence, it would be very useful if one could calculate the diffusion coefficients theoretically at any desired level in the atmosphere as a function of both temperature and pressure instead of adopting arbitrarily prescribed values.

In the next section we show the use of a 6-12 Lennard-Jones model to obtain the binary diffusivity of water vapour in air. Although the method is described in Ghosh (1993) and Ghosh et al. (1995) we outline the general procedure for the sake of completeness. In this study however, we have extended the original routines described in Ghosh (1993) and Ghosh et al. (1995) to account for the dipole moment of water, since $\mathrm{H}_{2} \mathrm{O}$ is a strongly polar molecule with a dipole moment of 1.8 Debyes.

\subsection{The Lennard-Jones method}

The theory describing diffusion in binary gas mixtures at low to moderate pressure has been well developed and the results are credited to Chapman and Enskog. Simplified versions can be found in standard textbooks of physical chemistry (e.g. Maitland et al., 1982). Based on the kinetic theory, the diffusion coefficient may be expressed as (Reid et al., 1987):

$D_{A B}=\frac{0.00266 T^{3 / 2}}{P M_{A B}^{1 / 2} \sigma_{A B}^{2} \Omega_{D}}$,

where,

$M_{A}, M_{B}=$ molecular weights of $A$ and $B$,

$M_{A B}=2\left[\left(1 / M_{A}+1 / M_{B}\right)\right]^{-1}$,

$T=$ temperature, $\mathrm{K}$,

$P=$ pressure, bar,

$\sigma_{A B}=$ characteristic length, $\AA$,

$\Omega_{D}=$ diffusion collision integral, dimensionless,

$D_{A B}=$ diffusion coefficient, $\mathrm{cm}^{2} \mathrm{~s}^{-1}$.

The key to the use of Eq. (30) is the selection of an intermolecular force law and the evaluation of $\Omega_{D}$. Reid and Sherwood (1958) emphasise that of all the available methods for correlating the intermolecular energy $\psi$ between two molecules to the distance of separation $r$, the most accurate method is that given by Lennard-Jones:

$\psi=4 \epsilon\left[\left(\frac{\sigma}{r}\right)^{12}-\left(\frac{\sigma}{r}\right)^{6}\right]$

with $\epsilon$ and $\sigma$ as the characteristic Lennard-Jones energy and length, respectively. To use Eq. (30) some rule must be chosen to obtain $\sigma_{A B}$ from $\sigma_{A}$ and $\sigma_{B}$. Also, it can be shown that $\Omega_{D}$ is a function only of $k T / \epsilon_{A B}$ ( $k$ being Boltzmann's constant), where again some rule must be selected to relate $\epsilon_{A B}$ to $\epsilon_{A}$ and $\epsilon_{B}$. The following simple rules are usually employed:

$\epsilon_{A B}=\left(\epsilon_{A} \epsilon_{B}\right)^{1 / 2}$,

$\sigma_{A B}=\left(\sigma_{A}+\sigma_{B}\right) / 2$.

$\Omega_{D}$ is tabulated as a function of $k T / \epsilon$ for the 6-12 Lennard-Jones potential and various analytical approximations are also available. We used the relation of Neufeld et al. (1972):

$\Omega_{D}=\frac{A}{\left(T^{*}\right)^{B}}+\frac{C}{\exp \left(D T^{*}\right)}+\frac{E}{\exp \left(F T^{*}\right)}+\frac{G}{\exp \left(H T^{*}\right)}$,

where,

$T^{*}=k T / \epsilon_{A B}, A=1.06036, B=0.15610, C=0.19300$,

$D=0.47635, E=1.03587, F=1.52996, G=1.76474$ and $H=3.89411$.

Application of the Chapman-Enskog theory to the viscosity of pure gases has led to the determination of many values of $\epsilon$ and $\sigma$ (Reid et al., 1987; Bzowski et al., 1990).

Polar gases. If one or both components of a gas mixture are polar, a modified Lennard-Jones relation, such as 
the Stockmayer potential, is often used. A different collision integral relation becomes necessary and Lennard-Jones $\sigma$ and $\epsilon$ values are not sufficient (Reid et al., 1987).

A modified collision integral $\Omega_{D}$ is now given as (Reid et al., 1987)

$\Omega_{D}=\Omega_{D}\left[\right.$ Eq.(34)] $+\frac{0.19 \delta_{A B}^{2}}{T^{*}}$,

where $T^{*}=\frac{k T}{\epsilon_{A B}}$

and $\delta=\frac{1.94 \times 10^{3} \mu_{p}^{2}}{V_{b} T_{b}}$

$\mu_{p}=$ dipole moment, debyes,

$V_{b}=$ liquid molar volume at the normal boiling point, $\mathrm{cm}^{3} \mathrm{~mol}^{-1}$,

$T_{b}=$ normal boiling point $(1 \mathrm{~atm}), \mathrm{K}$

$\frac{\epsilon}{k}=1.18\left(1+1.3 \delta^{2}\right) T_{b}$,

$\sigma=\left(\frac{1.585 V_{b}}{1+1.3 \delta^{2}}\right)^{1 / 3}$

$\delta_{A B}=\left(\delta_{A} \delta_{B}\right)^{1 / 2}$,

$\frac{\epsilon_{A B}}{k}=\left(\frac{\epsilon_{A}}{k} \frac{\epsilon_{B}}{k}\right)^{1 / 2}$

$\sigma_{A B}=\left(\sigma_{A} \sigma_{B}\right)^{1 / 2}$.

For $\mathrm{H}_{2} \mathrm{O}, V_{b}=18.7 \mathrm{~cm}^{3} \mathrm{~mol}^{-1}, T_{b}=375.15 \mathrm{~K}$; and using the foregoing relations we find that $\delta_{\mathrm{H}_{2} \mathrm{O}}=0.9$, $\left(\frac{\epsilon}{k}\right)_{\mathrm{H}_{2} \mathrm{O}}=903.97 \mathrm{~K}$ and $\sigma_{\mathrm{H}_{2} \mathrm{O}}=2.43 \AA$.

\subsection{Re-evaluation of the droplet evaporation term}

Using the preceding formulation we are able to calculate a typical diffusivity profile of water vapour. In order to do this we needed temperature and pressure profiles. We used the U.K. Meteorological Office LES model constrained with EUCREX temperature observations. This is shown in Fig. 5, from which we clearly find that the diffusion coefficient of water vapour between 900 $1200 \mathrm{~m}$ (a typical region where the stratocumulus clouds are observed) is $0.2 \mathrm{~cm}^{2} \mathrm{~s}^{-1}$. In the same figure we have also shown the temperature profile.

Having obtained the diffusivity profile for water vapour we shall now compare our estimation of the term $D_{v}\left(\rho_{a}-\rho_{b}\right)$ using Eq. (29) instead of Eq. (27) with those of Kinzer and Gunn (1951) and Beard and Pruppacher (1971). These authors report their experimental values of their estimation of $D_{v}\left(\rho_{a}-\rho_{b}\right)$ at a pressure of one atmosphere for varying relative humidities as well as temperatures. We have used their results for a temperature of $0{ }^{\circ} \mathrm{C}$ corresponding to the conditions relevant to the stratocumulus cloud simulated by the LES code. This is shown in Fig. 6, where the results of the Kessler (1969) scheme are also shown. We observe excellent agreement between the observations of Beard and Pruppacher (1971) and the present approach. We find that whereas in the Kessler parameterisation one

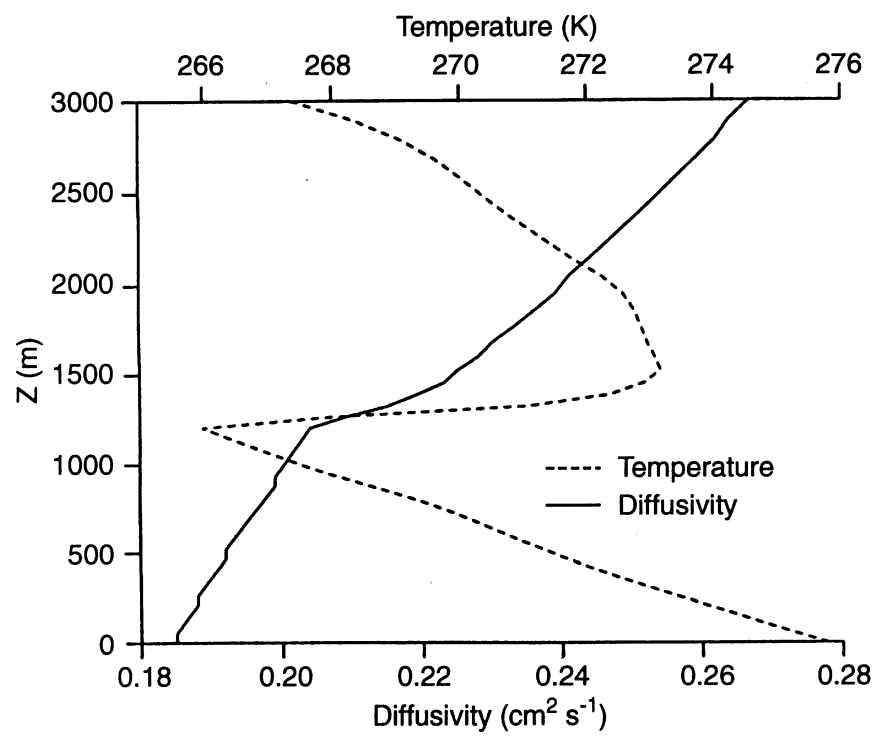

Fig. 5. Calculated diffusivity profile of $\mathrm{H}_{2} \mathrm{O}$ using the Lennard-Jones model and the temperature profile from the LES model

observes an overall underprediction, in our analysis we have much better agreement at all humidity levels.

Unfortunately, there are no experimental data available for a comparison also at lower temperatures (e.g. at $\left.-10^{\circ} \mathrm{C}\right)$.

The analysis in this section clearly points to the importance of aiming towards accurate mass transport estimates involved in cloud microphysical processes, and in particular this study demonstrates the usefulness of the Lennard-Jones model for an accurate estimation of the diffusion coefficient - a coefficient that one frequently encounters in mass-transfer calculations.

\section{Conclusions}

In this paper we have carefully examined the process of cloud autoconversion as described in the classic Kessler

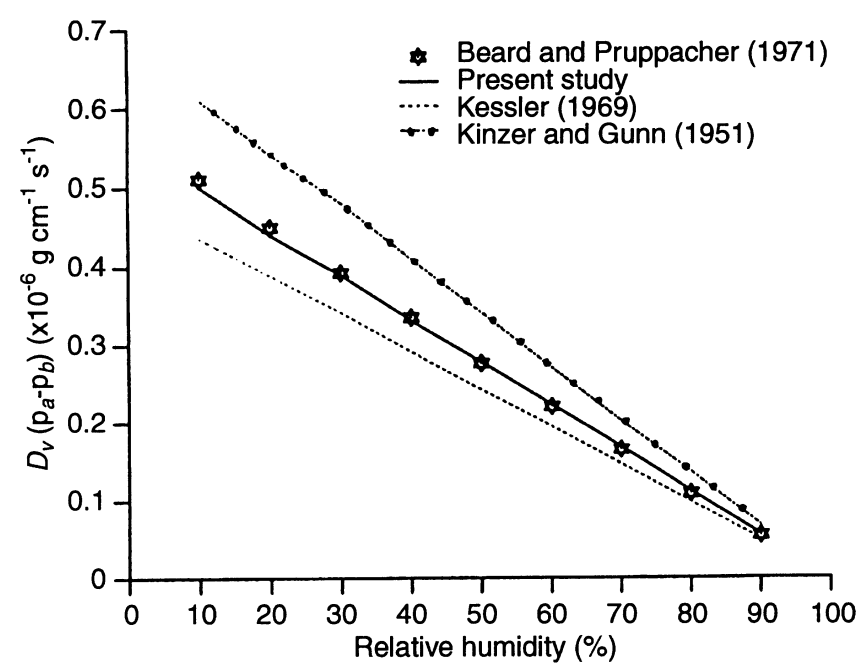

Fig. 6. $D_{v}\left(\rho_{a}-\rho_{p}\right)$ as a function of relative humidity 
(1969) and Berry (1968) schemes. We discuss in detail the applicability of these schemes in LES models. We are also able to derive new analytical expressions for the time of onset of precipitation using both the Kessler and Berry formulations. We then discuss the conditions when the two schemes are equivalent. Our study demonstrates that by first calculating the autoconversion coefficient as a function of droplet number density and droplet dispersion using the Berry (1968) scheme, one can then calculate the autoconversion threshold rather than arbitrarily specify two adjustable parameters in the Kessler formula. In this way one can still use the simple and linear Kessler formula in LES models and also indirectly incorporate the effects of different cloud types via the Berry (1968) formulation as described.

Finally, we also critically examine the process of droplet evaporation within the framework of the classic Kessler scheme. We improve the existing parameterisation with an accurate estimation of the diffusional mass transport of water vapour. We then demonstrate the overall robustness of our calculations by comparing our results with the experimental observations of Beard and Pruppacher (1971). This comparison shows an excellent agreement.

Acknowledgements. J. P. Duvel thanks T. Trautmann and another referee for their help in evaluating this paper.

\section{References}

Beard, K. V., and H. R. Pruppacher, A wind tunnel investigation of the rate of evaporation of small water drops falling at terminal velocity in air, J. Atmos. Sci., 28, 1455-1464, 1971.

Berry, E. X., Modification of the warm rain process, Proc. First Natl. Conf. Weather modification, Ed. American Meteorological Society, State University of New York, Albany, pp. 81-88, 1968.

Bzowski, J., J. Kestin, E. A. Mason, and F. J. Uribe, Equilibrium and transport properties of gas mixtures at low density: eleven polyatomic gases and five noble gases, J. Phys. Chem. Ref. Data, 19, 1179-1232, 1990.

Derbyshire, S. H., A. R. Brown, and A. P. Lock, The meteorological office large eddy simulation model. Met $O$ (APR) Turbulence and Diffusion Note, No., 213, Bracknell 1994.

Ghosh, S., On the diffusivity of trace gases under stratospheric conditions, J. Atmos. Chem., 17, 391-397, 1993.

Ghosh, S., D. J. Lary, and J. A. Pyle, Estimation of heterogeneous reaction rates for stratospheric trace gases with a particular reference to the diffusional uptake of $\mathrm{HCl}, \mathrm{ClONO}_{2}, \mathrm{HNO}_{3}$, and $\mathrm{N}_{2} \mathrm{O}_{5}$ by polar stratospheric clouds, Ann. Geophysicae, 13, 406-412, 1995.

Jonas, P. R., and S. Ghosh, Simulation of a stratocumulus topped planetary boundary layer with a particular emphasis on the modes of energy transfer between updraughts and downdraughts. To appear in a EUCREX special issue, Toulouse, France, 1997.

Jonas, P. R., and B. J. Mason, The evolution of droplet spectra by condensation and coalescence in cumulus clouds. $Q$. J. $R$. Meteorol. Soc., 100, 286-295.

Kessler, E., On the distribution and continuity of water substance on atmospheric circulation. Meteorol. Monogr., 10 (32), 84, 1969.

Kinzer, G. D., and R. Gunn, The evaporation, temperature and thermal relaxation time of freely falling water drops. $J$. Meteorol., 8, 71-83, 1951.

Maitland, G. C., M. Rigby, E. B. Smith, and W. A. Wakeham, Intermolecular forces - their origin and determination, Oxford Science Publications, Oxford, 1982.

Mason, B. J., The physics of clouds, Clarenden Press, Oxford, 1957.

Neufeld, P. J., A. R. Jansen, and R. A. Aziz, Empirical equations to calculate 16 of the transport collision integrals $\Omega^{(l, s) *}$ for the Lennard-Jones (12-6) potential, J. Chem. Phys., 57, 1100-1102, 1972.

Pawlowska, H., and J. L. Brenguier, A study of the microphysical structure of stratocumulus clouds. Proc. 12th Int. Conf. Clouds and precipitation, Zurich, Ed. P. R. Jones, Published by Page Bros., Norwich, U.K., Vol. I, pp. 123-126, 1996.

Reid, R. C., and T. K. Sherwood, The properties of gases and liquids, McGraw-Hill, New York, 1958.

Reid, R. C., J. M. Prausnitz, and B. E. Poling, The properties of gases and liquids, McGraw-Hill, New York, 1987.

Rogers, R. R., and M. K. Yau, A short course in cloud physics, Pergamon Press, Oxford, 1989.

Rutledge, S. A., and P. V. Hobbs, The mesoscale and microscale structure and organisation of clouds and precipitation in midlatitude cyclones. VIII: a model for the "seeder-Feeder" process in warm-frontal rainbands, J. Atmos. Sci., 40, 11851206, 1983.

Simpson, J., and V. Wiggert, Models of precipitating cumulus towers, Mon. Weather Rev., 97, 471-489, 1969.

Singleton, F., and D. J. Smith, Some observations of drop-size distributions in low-layer clouds. Q. J. R. Meteorol. Soc., 86, 454-467, 1960.

Smithsonian Meteorological Tables, The Smithsonian Institution, Washington, D.C., 1958.

Warner, J., The microstructure of cumulus cloud. Pt. 2. the effect of droplet size distribution of the cloud nucleus spectrum and updraft velocity, J. Atmos. Sci., 26, 1272-1282, 1969.

Weinstein, A. I., A numerical model of cumulus dynamics and microphysics, J. Atmos. Sci., 27, 246-255, 1970. 